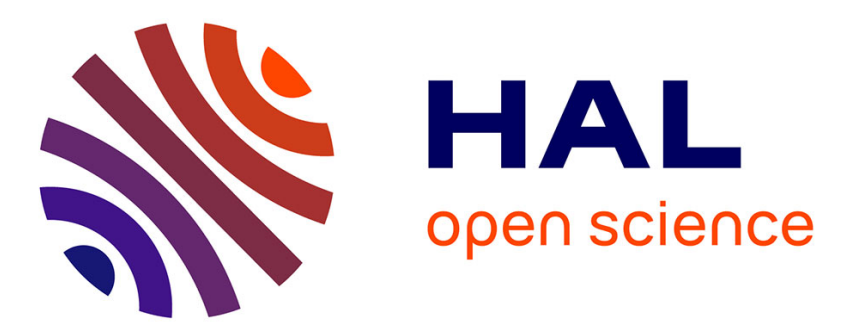

\title{
Mouvement d'une couche de fluide dissipatif en espace clos sous l'action d'une source étendue
}

\author{
P. Hamery, M. Bruneau, A.-M. Bruneau
}

\section{To cite this version:}

P. Hamery, M. Bruneau, A.-M. Bruneau. Mouvement d'une couche de fluide dissipatif en espace clos sous l'action d'une source étendue. Journal de Physique IV Proceedings, 1994, 04 (C5), pp.C5-213C5-216. 10.1051/jp4:1994541 . jpa-00253036

\section{HAL Id: jpa-00253036 https://hal.science/jpa-00253036}

Submitted on 1 Jan 1994

HAL is a multi-disciplinary open access archive for the deposit and dissemination of scientific research documents, whether they are published or not. The documents may come from teaching and research institutions in France or abroad, or from public or private research centers.
L'archive ouverte pluridisciplinaire HAL, est destinée au dépôt et à la diffusion de documents scientifiques de niveau recherche, publiés ou non, émanant des établissements d'enseignement et de recherche français ou étrangers, des laboratoires publics ou privés. 


\title{
Mouvement d'une couche de fluide dissipatif en espace clos sous l'action d'une source étendue
}

\author{
P. HAMERY, M. BRUNEAU et A.-M. BRUNEAU \\ Laboratoire d'Acoustique, URA 1101 du CNRS, Université du Maine, Avenue Olivier Messiaen, BP. 535, \\ 72017 Le Mans cedex, France
}

\begin{abstract}
Résumé: In recent years, the growing demand for miniaturized transducers and the widespread use of silicon technology, has focused interest on the design of condenser or electret microphones on a silicon chip. But the theoretical models used are those existing for conventional microphones, which do not remain valid for miniaturized transducers, if good accuracy is expected. The main purpose of the paper is to consider the forced vibrations of the membranes (or plates), driven by an external source (here an incident acoustic pressure field), backed by a very thin layer of fluid and a rigid plane wall, and surrounded by a reservoir around its periphery. The "exact" statement of the problem is obtained by taking into account effects of heat conduction, bulk and shear viscosity, impedance of the surrounding cavity, and so on, avoiding the approximations currently in use. An improved analytical solution is obtained and some new features are indicated.
\end{abstract}

\section{INTRODUCTION.}

Depuis une décennie environ, la demande croissante de transducteurs miniatures et l'utilisation des technologies sur silicium [1], les exigences liées à de nouveaux usages de ce type de transducteurs, de même que les études des propriétés dissipatives de couches de fluide dans diverses applications (amortissement des ondes dans les matériaux poreux, amortissement des vibrations de paliers, coussinets ou panneau, etc ...), on fait apparaître le besoin de modèles adaptés qui prennent en compte limportance relative des couches limites visqueuses et thermiques sur le mouvement du fluide emprisonné, le paramètre clé n'étant autre que l'épaisseur des couches limites mentionnées. Ainsi les vibrations forcées d'une fine couche de fluide, excitée par une source étendue et fermée par un volume clos à sa périphérie, font l'objet de cette étude : le problème "exact" est pris en considération, les hypothèses simplificatrices couramment admises n'étant pas retenues du fait qu'elles ne sont plus acceptables pour les raisons indiquées précédemment. La solution générale de ce problème est donnée, qui ouvre des perspectives nouvelles du fait qu'elle n'admet plus les approximations en usage jusqu'à maintenant. Des résultats portant pour l'instant sur le comportement hautes fréquences des transducteurs et sur les modèles à constantes localisées sont finalement présentés. 


\section{EQUATIONS DE BASE, CONDITIONS AUX FRONTIERES.}

Les modèles disponibles dans la littérature ([2] à [5]) prennent en compte l'inertie, la compressibilité et la viscosité de cisaillement du fluide. Mais ils admettent diverses hypothèses : la vitesse tourbillonnaire n'est pas complètement prise en compte et la viscosité de volume est négligée, la conduction thermique est le plus souvent supposée nulle et la vitesse laminaire associée est toujours négligée, le champ de pression suivant la normale à la membrane est supposée uniforme et la composante suivant cette normale de la vitesse particulaire nulle, la condition à la frontière périphérique pour la pression dans le fluide exclu les effets de viscosité et de conduction thermique (quand elle n'est pas celle de Dirichlet, alors même que l'espace est clos).

Aussi l'objet du travail présenté ici est-il de proposer un modèle théorique qui n'admet plus de telles hypothèses, dans lequel le paramètre clé n'est autre que l'épaisseur de la couche de fluide enfermée entre la membrane et l'électrode arrière, qui devient du même ordre de grandeur que l'épaisseur des couches limites visqueuses et thermiques dans les transducteurs miniatures. Le système d'équations linéarisées sur lequel repose l'étude du mouvement de la lame du fluide comprend pour l'essentiel l'équation de Stokes-Navier, l'équation de conservation de la masse et l'équation de conduction de la chaleur. Ecrivant la vitesse particulaire sous la forme de la superposition d'une vitesse tourbillonnaire $\vec{v}_{v}$ et d'une vitesse laminaire $\vec{v}_{\ell}\left(\vec{v}=\vec{v}_{\ell}+\vec{v}_{v}\right)$, ces équations prennent la forme suivante [6]:

$$
\begin{aligned}
& \partial_{\mathrm{ct}} \tau-\frac{\rho \mathrm{c}}{\gamma \beta} \operatorname{div}\left(\overrightarrow{\mathrm{v}}_{\ell}\right)=\frac{1}{\beta} \partial_{\mathrm{ct}} \mathrm{p}, \\
& \left(\partial_{\mathrm{ct}}-\ell_{\mathrm{h}} \Delta\right) \tau=\frac{\gamma-1}{\gamma \beta} \partial_{\mathrm{ct}} \mathrm{p}, \\
& \left(\partial_{\mathrm{ct}}-\ell_{\mathrm{v}} \Delta\right) \overrightarrow{\mathrm{v}}_{\ell}=-\frac{1}{\rho \mathrm{c}} \overrightarrow{\operatorname{grad}}(\mathrm{p}), \\
& \left(\partial_{\mathrm{ct}}-\ell_{\mathrm{v}}^{\prime} \Delta\right) \overrightarrow{\mathrm{v}}_{\mathrm{v}}=0,(4) \quad \operatorname{div}\left(\overrightarrow{\mathrm{v}}_{\mathrm{v}}\right)=0 \text { et } \overrightarrow{\operatorname{rot}}_{\mathrm{v}}\left(\overrightarrow{\mathrm{v}}_{\ell}\right)=\overrightarrow{0} \text {, }
\end{aligned}
$$

où $\tau$ représente la variation de température, $p$ la variation de pression, $\beta$ l'augmentation de pression isochore, $\gamma$ le rapport des capacités calorifiques à volume et à pression constante, et où $\ell_{\mathrm{v}}, \ell_{\mathrm{v}}^{\prime}, \ell_{\mathrm{h}}$ sont des longueurs caractéristiques fonctions des coefficients de viscosité de cisaillement $\mu$, de viscosité de volume $\eta$ et de conduction de la chaleur $\lambda$ :

$$
\ell_{\mathrm{v}}=[4 \mu / 3+\eta] / \rho \mathrm{c}, \ell_{\mathrm{v}}^{\prime}=\mu / \rho \mathrm{c}, \ell_{\mathrm{h}}=\lambda / \rho \mathrm{\rho C} \mathrm{C}_{\mathrm{p}} .
$$

Ces équations sont couplées à celles qui régissent le mouvement de la membrane, soumise à une onde de pression acoustique harmonique incidente supposée uniforme sur toute sa surface, et qui s'écrivent de la façon suivante :

$$
\begin{aligned}
& \mathrm{T}\left(\Delta+\mathrm{K}^{2}\right) \xi(\overrightarrow{\mathrm{w}})=\mathrm{p}_{\mathrm{i}}-\mathrm{p}(\overrightarrow{\mathrm{w}}, \mathrm{h}), \forall \overrightarrow{\mathrm{w}} \\
& \xi\left(\overrightarrow{\mathrm{w}}_{\mathrm{s}}\right)=0, \text { sur les bords de la membrane, }
\end{aligned}
$$

avec $K^{2}=\omega^{2} \mu_{s} / T$, où $\mathrm{T}$ est la tension linéique de la membrane, $\mu_{\mathrm{s}}$ sa masse surfacique et $\omega$ la pulsation.

Les conditions aux frontières retenues sont celles d'usage courant : variation de température nulle en parois, continuité des composantes normales et tangentielles des vitesses particulaires totales aux interfaces fluide-solide aux parois, réaction de type impédance et loi polytropique à la périphérie du film de fluide.

\section{SOLUTION DU PROBLEME.}

L'équation de continuité pour la vitesse normale à l'interface entre la membrane et le fluide doit être utilisée pour décrire le couplage fort existant entre la membrane et le fluide. De plus, la 
solution de l'équation (6) est cherchée sous une forme permettant de bénéficier des propriétés d'orthogonalité des solutions $\psi_{\mathrm{n}}\left(\mathrm{k}_{\overline{\mathrm{w}} \mathrm{n}} \overrightarrow{\mathrm{w}}\right)$ du problème aux valeurs propres associé de Dirichlet. forme:

Par suite, la solution générale pour le déplacement $\xi(w)$ de la membrane s'écrit sous la

$$
\xi(w)=\sum_{n} \frac{1}{T\left(K^{2}-k_{\vec{w} n}^{2}\right)} \iint d \vec{w}_{0}\left[p_{i}-p\left(\vec{w}_{0}, h\right)\right] \psi_{n}\left(k_{\vec{w} n} \vec{w}_{0}\right) \psi_{n}\left(k_{\vec{w} n} \vec{w}\right),
$$

et puisque la continuité des vitesses doit être vérifiée pour toutes les valeurs de $\overrightarrow{\mathrm{w}}$ sur toute la surface de la membrane, la vitesse normale du fluide à l'interface $\mathrm{z}=\mathrm{h}$ doit s'écrire :

$$
v_{z}(\vec{w}, h)=\sum_{n} u_{n}(h) \Psi_{n}\left(k_{\vec{w} n} \vec{w}\right)
$$

où $u_{n}(h)$ sont des coefficients et où $z$ est la coordonnée normale à la membrane et à l'électrode arrière rigide, situées respectivement en $z=h$ et $z=0$. Par prolongement, pour toute valeur de $z$, cette vitesse peut s'écrire :

$$
\mathrm{v}_{\mathrm{z}}(\overrightarrow{\mathrm{w}}, \mathrm{z})=\sum_{\mathrm{n}}\left[\mathrm{u}_{\mathrm{n}}(\mathrm{z}) \psi_{\mathrm{n}}\left(\mathrm{k}_{\overline{\mathrm{w}} \mathrm{n}} \overrightarrow{\mathrm{w}}\right)+\mathrm{v}_{\mathrm{n}}(\mathrm{w}) \phi_{\mathrm{n}}\left(\mathrm{k}_{\mathrm{zm}} \mathrm{z}\right)\right]
$$

où les fonctions $\phi_{\mathrm{n}}\left(\mathrm{k}_{\mathrm{m}} \mathrm{z}\right)$ doivent vérifier des conditions de Dirichlet en $\mathrm{z}=\mathrm{h}$.

Le premier développement, celui sur les fonctions $\psi_{n}\left(k_{\vec{\psi} n} \vec{w}\right)$, est la solution du problème nonhomogène correspondant au "mouvement forcé". Le deuxième développement, celui sur les fonctions $\phi_{\mathrm{n}}\left(\mathrm{k}_{\mathrm{z}} \mathrm{z}\right)$, correspond à la solution du "problème homogène associé".

Le potentiel des vitesses $q(\vec{w}, z)$ est obtenu à partir de l'équation (9) sous la forme :

$$
\mathrm{q}(\overrightarrow{\mathrm{w}}, \mathrm{z})=\sum_{\mathrm{n}}\left[\mathrm{q}_{\mathrm{an}}(\overrightarrow{\mathrm{w}}, \mathrm{z})+\mathrm{q}_{\mathrm{hn}}(\overrightarrow{\mathrm{w}}, \mathrm{z})\right]
$$

Dans ce résultat, les fonctions $\mathrm{q}_{\mathrm{an}}$ et $\mathrm{q}_{\mathrm{hn}}$ contiennent les parties acoustiques et entropiques des "coefficients" $u_{n}(z)$ et $v_{n}(w)$, et sont directement obtenues à partir des équations de propagation équivalentes au système d'équations (1) à (5).

De la même manière, il est aisé d'obtenir à partir des équations (4) et (9), une expression de la composante suivant $z$ de la vitesse tourbillonnaire $\vec{v}_{v}$.

La solution générale du problème pour chaque variable d'intérêt peut alors être obtenue en faisant usage des formes de solutions pour $q(\vec{w}, z)$ obtenu précédemment, formes adaptées à la géométrie du système considéré. Par exemple, pour la variation de température, la solution de l'équation non homogène (2), qui prend en compte le résultat $(10)$, s'écrit :

$$
\tau(\vec{w}, z)=-\frac{i \rho c}{k \gamma \beta} \sum_{n}\left[\alpha_{a} k_{a}^{2} q_{a n}(\vec{w}, z)+\alpha_{h} k_{h}^{2} q_{h n}(\vec{w}, z)\right]
$$

où $\alpha_{\mathrm{a}, \mathrm{h}}=(\gamma-1) /\left[1-\mathrm{i} \gamma \ell_{\mathrm{h}} \mathrm{k}_{\mathrm{a}, \mathrm{h}}^{2} / \mathrm{k}\right], \mathrm{k}=\omega / \mathrm{c}$ et où $\mathrm{k}_{\mathrm{a}}$ et $\mathrm{k}_{\mathrm{h}}$ représentent les nombres d'onde associés respectivement aux mouvements acoustique et entropique.

\section{RESULTATS ET CONCLUSIONS.}

L'impédance de la membrane chargée par le film de fluide peut être écrite sous la forme :

$$
Y=\frac{U}{P_{i}}=\sum_{n} \frac{A_{n}+\sum_{m} \alpha_{n m}}{\left(K^{2}-k_{\overrightarrow{\mathrm{w}} n}^{2}-\chi_{n}^{2}\right)}
$$

Dans cette expression, le facteur de couplage $\sum_{\mathbf{m}} \alpha_{n m}$ traduit l'effet de la réaction du volume périphérique à son interface avec la couche de fluide; ce facteur s'annule pour une condition de 
Dirichlet à sa périphérie. Sa présence est liée à celle du second développement de la solution (solution du problème homogène). La composante transversale $\mathrm{k}_{\mathrm{e} \tilde{\mathrm{m} m}}=\sqrt{\mathrm{k}_{\mathrm{a}}^{2}-(\mathrm{m} \pi / \mathrm{h})^{2}}$ du nombre d'onde acoustique $\mathrm{k}_{\mathrm{a}}$, associé à ce développement vaut $\mathrm{k}_{\mathrm{a}}$ lui même pour $\mathrm{m}=0$ (valeur finie à partie imaginaire très petite), et se comporte comme i $m \pi / h$ pour $m \neq 0$ valeur très grande (car $h$ est très petit) et imaginaire pure, qui correspond à un phénomène évanescent (du centre du système vers la périphérie). On vérifie de la sorte que le facteur $m=0$ est prépondérant; c'est le seul terme que nous retenons dans ce développement dans la suite du calcul, conservant par ailleurs les modes de membrane $\psi_{\mathrm{n}}\left(\mathrm{k}_{\overrightarrow{\mathrm{y}} \mathrm{x}} \overrightarrow{\mathrm{w}}\right)$ d'ordres élevés. A titre d'exemple, la figure 1 montre les résultats obtenus pour la sensibilité d'un transducteur microphonique électrostatique, $\sigma=Y . E /(j \omega V)$ (E est la différence de potentiel entre les électrodes et $V$ le volume de la lame d'air), dont les caractéristiques géométriques sont données dans la légende de la figure. La figure 2 montre un circuit à constantes localisées obtenu en négligeant les effets liés au facteur $\sum_{m} \alpha_{\mathrm{nm}}$; on remarque que ce circuit diffère notablement de ceux qui sont d'usage courant [5].
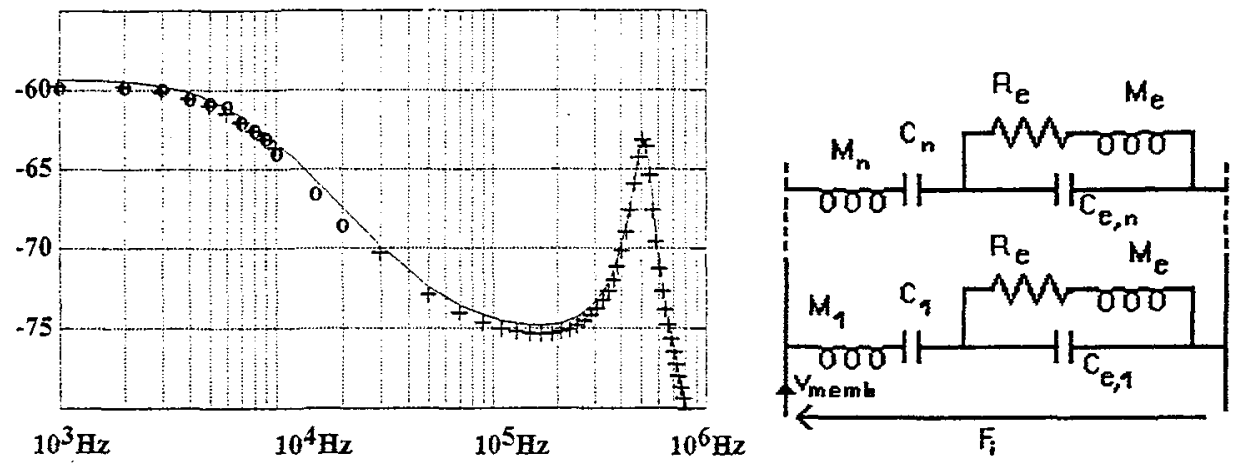

figure 1 : Sensibilité d'un microphone en $\mathrm{dB}(\mathrm{ref}: \mathrm{V} / \mathrm{Pa})$,

- théorie complète,

+ circuit équivalent,

- expérience,

figure 2 : Circuit équivalent où :

$M_{n}=\left(\gamma_{n}^{2} / 4\right)_{s} \pi R^{2}, C_{n}=4 /\left(\gamma_{n}^{4} \pi T\right)$.

$\mathrm{R}_{\mathrm{e}}=3 \rho c \ell_{\mathrm{v}}^{\prime} \pi \mathrm{R}^{4} / \mathrm{h}^{3}, \mathrm{M}_{\mathrm{e}}=3 \rho \pi \mathrm{R}^{4} /(8 \mathrm{~h})$,

$\mathrm{C}_{\mathrm{c}, \mathrm{n}}=\zeta_{\mathrm{n}} \mathrm{h} /\left(\rho \mathrm{c}^{2} \pi \mathrm{R}^{2}\right), \mathrm{J}_{0}\left(\gamma_{\mathrm{n}}\right)=0$,

(entrefer: $\mathrm{h}=510^{-6} \mathrm{~m}$, surface: $\mathrm{S}=0.510^{-6} \mathrm{~m}^{2}$,

polarisation: $E=20 \mathrm{~V}$, volume arrière: $1.5 \mathrm{e}^{-10} \mathrm{~m}^{3}$ ).

$\zeta_{\mathrm{D}}=\left(4 / \gamma_{\mathrm{n}}^{2}\right) \frac{\left[\gamma+(\gamma+2)\left(j \omega^{2} / 24 \mathrm{c} \ell_{\mathrm{h}}\right)\right]}{\left[1+\left(j \omega^{2} / 8 \mathrm{c} \ell_{\mathrm{b}}\right)\right]}$.

[1] HOHM.D.,HESS.G.,A subminiature condenser microphone with silicon nitride membrane and silicon back-plate,J.Acoust.Soc.Am., 85(1989), 476-480.

[2] ROBEY.D.H., Theory of the effect of a thin air film on the vibrations of a stretched circular membrane,J.Acoust. Soc. Am., 26(1954), 740-745.

[3] WARREN.J.E.,BRZEZINSKI.A.M. and HAMILTON.J.F., Capacitance microphone dynamic membrane deflection,J.Acoust.Soc.Am.,54,(5),(1973), 1201-1213.

[4] ZUCKERWAR.A.J., Theoretical response of condenser microphones,J.Acoust.Soc.Am., 64,(5), (1978), 1278-1285.

[5] SKVOR.Z., Vibrating systems and their equivalent circuits,(Elsevier Science Publishers, Ansterdam, Oxford,New York, Tokyo, 1991).

[6] BRUNEAU.M.,HERZOG.P.H.,KERGOMARD.J.,POLACK.J.-D.,General formulation of the dispersion equation in bounded visco-thermal fluid, and application to some simple geometries, Wave Motion, 11,(1989),441-451. 